\title{
Reciprocal regulation of long noncoding RNAs THBS4-003 and THBS4 control migration and invasion in prostate cancer cell lines
}

\author{
JINLIANG LIU*, GONG CHENG* , HAIWEI YANG, XIAHENG DENG, \\ CHAO QIN, LIXIN HUA and CHANGJUN YIN \\ State Key Laboratory of Reproductive Medicine, Department of Urology, \\ The First Affiliated Hospital of Nanjing Medical University, Nanjing, \\ Jiangsu 210029, P.R. China
}

Received March 1, 2015; Accepted February 18, 2016

DOI: $10.3892 / \mathrm{mmr} .2016 .5443$

\begin{abstract}
Increasing evidence implicates long noncoding RNAs (IncRNAs), a class of noncoding RNAs >200 nucleotides in length, in the development of cancer. However, the mechanism underlying the effects of IncRNAs in prostate cancer $(\mathrm{PCa})$ remains to be elucidated. The present study aimed to investigate the role of IncRNA-THBS4-003 in the pathogensis of PCa. In the present study, a microarray containing 8,277 lncRNA probes and 32,207 mRNA probes were used to identify dysregulated mRNAs in three patients with PCa, and reverse transcription-quantitative polymerase chain reaction was used to determine the expression levels of thrombospondin 4 (THBS4) and lncRNA-THBS4-003 in 46 primary $\mathrm{PCa}$ and adjacent non-tumor tissue samples. The expression levels of THBS4 were determined in six samples of PCa and adjacent non-tumor tissues using Western blot analysis. The effects of forced THBS4 knockdown and lncRNA-THBS4-003 knockdown in the two PCa cell lines, DU145 and PC-3, were evaluated using cell migration and invasion assays, as well as using Western blot analysis. Of the 40,484 probes in the microarray, 354 were significantly upregulated $(\mathrm{P}<0.05$; fold-change $>2)$. The most significantly upregulated mRNA was THBS4. The expression levels of THBS4 and 1ncRNA-THBS4-003 in the 46 primary $\mathrm{PCa}$ samples was significantly higher, compared with that in the adjacent non-tumor tissue samples. Patients with Gleason scores $>7$ exhibited higher expression levels of lncRNA-THBS4-003, compared with patients with lower scores. Knockdown of
\end{abstract}

Correspondence to: Dr Lixin Hua, State Key Laboratory of Reproductive Medicine, Department of Urology, The First Affiliated Hospital of Nanjing Medical University, 300 Guangzhou Road, Nanjing, Jiangsu 210029, P.R. China

E-mail: hua_lixin@163.com

* Contributed equally

Key words: long noncoding RNA, IncRNA-THBS4-003, prostate cancer, mitogen-activated protein kinase signaling pathway
THBS4 or lncRNA-THBS4-003 significantly reduced the migratory and invasive abilities of the PCa cells in vitro, and decreased the expression levels of p38 and matrix metalloproteinase (MMP)-9. These findings suggested that the reciprocal regulation of IncRNA-THBS4-003 and THBS4 contributed to the pathogenesis of $\mathrm{PCa}$. Therefore silencing lncRNA-THBS4-003 or THBS4 may inhibit PCa cell migration and invasion, and regulate the levels of MMP-9 through the mitogen-activated protein kinase signaling pathway.

\section{Introduction}

Prostate cancer ( $\mathrm{PCa}$ ) is one of the most frequently diagnosed malignancies in men worldwide (1). The incidence of $\mathrm{PCa}$ in China has increased $(2,3)$, however, the mechanisms underlying the development and progression of PCa remain to be fully elucidated. Disease relapse and metastasis, as well as the development of hormone-refractory disease, remain the leading causes of mortality. Therefore, investigating the molecular mechanisms involved in the progression of $\mathrm{PCa}$ is of major importance and may reveal novel approaches for targeted PCa therapy.

With the advent of tiling-resolution genomic microarrays and whole-genome and transcriptome sequencing technologies, studies have revealed that at least $90 \%$ of the genome is actively transcribed $(4,5)$. Several of these transcripts have emerged as critical regulators of gene expression and determination of cell fate. A recently identified group of transcripts, termed long noncoding RNAs (lncRNAs), may contribute to a significant quantity of non-coding RNA which makes up the human transcriptome $(6,7)$. IncRNAs are a class of noncoding RNAs $>200$ nucleotides in length; they can be transcribed by RNA polymerase (Pol) II/Pol I, and a number are transcribed by RNA Pol III. IncRNAs are involved in several biological processes, including $\mathrm{X}$ chromosome inactivation, nuclear structure, genomic imprinting and development $(8,9)$. IncRNA dysfunction has been associated with cell fate determination and the pathogenesis of human disease, including cancer (10). Furthermore, several dysregulated lncRNAs are associated with the carcinogenesis and growth of tumors, including breast cancer, colon cancer, PCa, hepatocellular carcinoma and leukemia (11-15). 
lncRNA-thrombospondin 4 (THBS4)-003 is a processed transcript, which does not encode a protein product and has a length of $558 \mathrm{bp}$, which is $>200 \mathrm{bp}$ and, thus fits well within the definition for IncRNAs. IncRNA-THBS4-003 is located at chromosome $5 \mathrm{p} 14.1$ and partially overlaps with the protein-coding gene, THBS4 (16).

Mitogen-activated protein kinases (MAPKs) are a family of kinases, which comprise one of the major signaling pathways involved throughout the development of PCa. MAPKs can be divided into three subfamilies: The extracellular-signal-regulated kinases, the c-Jun N-terminal kinases, and p38 MAPK. It is well known that p38 MAPK is capable of regulating several cellular responses to cytokines and stress; however, previous data demonstrated that p38 is also closely linked to the development of different types of human cancer, through its ability to enhance cancer cell migration and invasion (17). Matrix metalloproteinases (MMPs), members of the zinc-dependent endoprotease family, are important enzymes involved in degrading the dermal extracellular matrix (ECM); in particular, MMP-9 is important in cancer cell invasion and metastasis (18).

In the present study, THBS4 and lncRNA-THBS4-003 expression levels are analyzed in PCa tissue in comparison with normal prostate tissue samples, and its potential biomedical functions in vitro are investigated. The aim of the study is to confirm whether IncRNA-THBS4-003 is a potential therapeutic target due to its role in PCa migration and invasion.

\section{Materials and methods}

Ethical statement. The present study was approved by the Institutional Review Board of the First Affiliated Hospital of Nanjing Medical University (Nanjing, China). During recruitment, written informed consent was obtained from all participants involved in the present study.

Tissue collection. Primary PCa and adjacent non-tumor tissue samples were collected from patients undergoing radical prostatectomy between 2011 and 2013 at the Department of Urology, the First Affiliated Hospital of Nanjing Medical University. Neither local nor systemic treatment had been administered in these patients prior to surgery. A total of 46 samples from 46 patients undergoing radical prostatectomy were collected in this study. The size of samples were $0.5 \mathrm{~cm}^{3}$, at least containing $200 \mathrm{mg}$ cells. Following surgery, all the samples were immediately frozen and stored in liquid nitrogen until further analysis. Only samples containing $>70 \%$ tumor cells were used for the extraction of total RNA. All experiments were approved by the Research Ethics Committee of Nanjing Medical University,. Detailed information on each tissue donor is provided in Table I.

Bioinformatics microarray data analysis. The microarray contained 8,277 IncRNA probes, which were designed by Arraystar Human LncRNA Expression Microarray (version 4.0; Arraystar, Inc., Rockville, MA, USA), based on the RefSeq (http://www.ncbi.nlm.nih.gov/refseq/), UCSC Known genes (http://genome.ucsc.edu/), and Ensembl (http://ensemblgenomes.org/)databases and associated literature $(19,20) ; 32,207$ protein-coding transcripts were
Table I. Patient characteristics.

Number of $\mathrm{PCa}$

Characteristic samples $(n=46)$

$\begin{array}{lr}\text { Age (years) } & \\ \text { Median (range) } & \\ \text { T stage } & 6 \\ \text { T1 } & 22 \\ \text { T2 } & 10 \\ \text { T3 } & 8 \\ \text { T4 } & \\ \text { N stage } & 38 \\ \text { N0 } & 8 \\ \text { N1 } & \\ \text { M stage } & 46 \\ \text { M0 } & 0 \\ \text { M1 } & \\ \text { Gleason score } & \\ <7 & 13 \\ 7 & 20 \\ >7 & 13\end{array}$

PCa, prostate cancer; T, tumor; N, node; M, metastasis.

used for microarray assays in three PCa tissue samples and their matched non-tumor samples. Differentially expressed lncRNAs and mRNAs, found to be statistically significant $[\mathrm{P}<0.05$; fold-change $(\mathrm{FC})>2]$ between the two groups were identified by comparing the normalized expression levels in the tumor and non-tumor samples using a paired t-test. Hierarchical clustering was then performed to analyze the differential lncRNA and mRNA expression patterns.

Cell lines and cell culture. The DU145 and PC-3 human PCa cell lines were purchased from the Institute of Biochemistry and Cell Biology of the Chinese Academy of Sciences (Shanghai, China). The DU145 and PC-3 cells (60-70\% confluence) were cultured in F-12K (Gibco; Thermo Fisher Scientific, Inc., Waltham, MA, USA) supplemented with $10 \%$ fetal bovine serum (FBS), $100 \mathrm{U} / \mathrm{ml}$ penicillin and $100 \mathrm{mg} / \mathrm{ml}$ streptomycin (Gibco; Thermo Fisher Scientific, Inc.) at $37^{\circ} \mathrm{C}$ with $5 \% \mathrm{CO}_{2}$.

$R N A$ isolation and reverse transcription-quantitative polymerase chain reaction (RT-qPCR) analysis. For the analyses of mRNA, total RNA was extracted from $75 \mathrm{mg}$ tissue samples and cultured cells using $1 \mathrm{ml}$ TRIzol reagent (Invitrogen; Thermo Fisher Scientific, Inc.), according to the manufacturer's protocol, and centrifugation at $12,000 \mathrm{x} \mathrm{g} \mathrm{(15} \mathrm{min}$ at $\left.4^{\circ} \mathrm{C}\right)$. For RT-qPCR, $1 \mu \mathrm{g}$ total RNA was reverse transcribed into cDNA in a final volume of $20 \mu \mathrm{l}$, using random primers and a High Capacity RNA-to-cDNA kit (Applied Biosystems; Thermo Fisher Scientific, Inc.), according to the manufacturer's protocol. The RT reaction was performed at $37^{\circ} \mathrm{C}$ for $60 \mathrm{~min}$, followed by $95^{\circ} \mathrm{C}$ for $5 \mathrm{~min}$. The expression levels of 
lncRNA-THBS4-003 and THBS4 in the PCa cell lines and tissues were then measured using qPCR, according to the standard protocol of the SYBR Select Master mix (Applied Biosystems; Thermo Fisher Scientific, Inc.). The sequences of the PCR primers used were as follows: IncRNA-THBS4-003, forward 5'-ATGAAGGCTCTGAGTTGGTG-3' and reverse 5'-CTTGGAAGTCCTCAGGGATG-3'; THBS4, forward 5'-GTTGCAGAACCTGGCATTCAG-3' and reverse 5'-CCC TGGACCTGTCTTAGACTTCA-3'; and $\beta$-actin, forward 5'-ACTGGAACGGTGAAGGTGAC-3' and reverse 5'-AGA GAAGTGGGGTGGCTTTT-3'. Primers were synthesized by Invitrogen (Thermo Fisher Scientific, Inc.). The mRNA detection reaction was performed under the following conditions: $50^{\circ} \mathrm{C}$ for $2 \mathrm{~min}, 95^{\circ} \mathrm{C}$ for $2 \mathrm{~min}, 40$ cycles at $95^{\circ} \mathrm{C}$ for $15 \mathrm{sec}$, and $60^{\circ} \mathrm{C}$ for $1 \mathrm{~min}$. The expression levels of lncRNA-THBS4-003 and THBS4 were normalized to that of $\beta$-actin and calculated using the $\Delta \Delta^{\mathrm{Cq}}$ method (21). The reactions were performed and analyzed using an ABI StepOne plus system (Applied Biosystems; Thermo Fisher Scientific, Inc.). All reactions were run in triplicate.

Small interfering (si)RNA transfection. Briefly, $1 \times 10^{5}$ cells were seeded into six-well plates and cultured in complete growth media at $37^{\circ} \mathrm{C}$ until the cell density reached $60-70 \%$, prior to siRNA transfection using Lipofectamine 2000 (Thermo Fisher Scientific, Inc.), according to the manufacturer's protocol. The cells were harvested after $48 \mathrm{~h}$ at $37^{\circ} \mathrm{C}$ for RT-qPCR and Western blot analyses. The sequences of the siRNAs used in the present study were as follows: 5'-GGC AACAGCUACAGUACAATT-3' and 5'-UUGUACUGUAGC UGUUGCCTT-3' for si-lncRNA-THBS4-003, 5'-GGCAGU UCUUGGGUCAAAUTT-3' and 5'-AUUUGACCCAAGAAC UGCCTT-3' for si-THBS4, and 5'-UUCUCCGAACGUGUC ACGUTT-3' and 5'-ACGUGACACGUUCGGAGAATT-3' for the control siRNA (Invitrogen; Thermo Fisher Scientific, Inc.).

Cell migration and invasion assays. For the migration assays, $3 \times 10^{4}$ cells in serum-free medium were placed in the upper chamber of a Transwell (pore size, $8 \mathrm{~mm}$; BD Biosciences, San Jose, CA, USA). For the invasion assays, cells with $200 \mu \mathrm{l}$ serum-free medium were placed in upper chambers coated with Matrigel (BD Biosciences), according to the manufacturer's protocol. Medium containing 10\% FBS was immediately added to the lower chamber as a chemoattractant. Following incubation for $24 \mathrm{~h}$ at $37^{\circ} \mathrm{C}$, the cells, which had not migrated through the pores of the Transwell inserts were manually removed using a cotton swab, and those cells on the lower surface of the membrane were fixed in $95 \%$ ethanol and stained with $0.1 \%$ crystal violet (Beyotime Institute of Biotechnology, Haimen, China). The numbers of cells in five randomly-selected fields were determined for each chamber, and the average value was calculated. Each experiment was performed in triplicate. Matrigel invasion assays were performed, as described previously (22).

Protein isolation and Western blot analysis. The tissue samples and cells were washed twice in ice-cold phosphate-buffered saline and then lysed in buffer containing $20 \mathrm{mM}$ Tris- $\mathrm{HCl}$ (pH 7.4; Bio-Rad Laboratories, Inc., Hercules, CA, USA), $137 \mathrm{mM} \mathrm{NaCl}, 10 \%$ glycerol, 1\% Triton X-100, 2 mM EDTA,
$25 \mathrm{mM} \beta$-glycerophosphate, $2 \mathrm{mM}$ sodium pyrophosphate and $0.5 \mathrm{mM}$ dithiothreitol (all purchased from Sigma-Aldrich, St. Louis, MO, USA) with protease inhibitors at $4^{\circ} \mathrm{C}$ for $30 \mathrm{~min}$. Subsequently, cellular debris was removed by centrifugation of the lysate at $12,000 \times \mathrm{g}$ for $10 \mathrm{~min}$ at $4^{\circ} \mathrm{C}$. The supernatants $(50 \mu \mathrm{l})$ were mixed with equal volumes of $2 \mathrm{X}$ sodium dodecyl sulfate (SDS) sample buffer (Sigma-Aldrich) and heated to $100^{\circ} \mathrm{C}$ for $10 \mathrm{~min}$. An equal volume of sample was fractionated by SDS-PAGE on a $10 \%$ acrylamide gel and transferred onto polyvinylidene difluoride membranes using a Bio-Rad transfer system (version 4.62) (both purchased from Bio-Rad Laboratories, Inc.), according to the manufacturer's protocol. Following blocking of the non-specific binding sites with 5\% nonfat milk in Tris-buffered saline with Tween 20, containing $50 \mathrm{mM}$ Tris, $0.15 \mathrm{M} \mathrm{NaCl}$ and $0.1 \%$ Tween 20 (pH 7.6) for $1 \mathrm{~h}$, the membranes were probed with THBS4 rabbit polyclonal antibody (1:2,000 dilution; cat. no. ab121094; Abcam, Cambridge, MA, USA), p38 rabbit polyclonal antibody (1:1,000 dilution; cat. no. ab7952; Abcam), rabbit polyclonal anti-MMP-9 antibody (1:1,000 dilution; cat. no. ab38898; Abcam), or $\beta$-actin rabbit antibody (1:1,000 dilution; cat. no. 8457; Cell Signaling Technology, Inc., Danvers, MA, USA) at $4{ }^{\circ} \mathrm{C}$ overnight, followed by incubation with horseradish peroxidase-conjugated goat anti-rabbit secondary antibodies (1:1,000 dilution; cat. no. 7074; Cell Signaling Technology, Inc.) for $1 \mathrm{~h}$ at $25^{\circ} \mathrm{C}$. Immunoreactive bands were visualized using enhanced chemiluminescence reagents (Thermo Fisher Scientific, Inc.), according to the manufacturer's protocol. Densitometric analysis of the immunoblots was performed using QuantityOne software (version 4.62; Bio-Rad Laboratories, Inc.). Protein levels were determined by normalization to $\beta$-actin, and the mean \pm standard deviation was calculated from three individual experiments.

Statistical analysis. The results are presented as the mean \pm standard error of the mean. Differences between groups were assessed for significance using Student's $t$-test. $\mathrm{P}<0.05$ was considered to indicate a statistically significant difference. All statistical analyses were performed using SPSS 11.0 for Windows (SPSS Inc, Chicago, IL, USA).

\section{Results}

Expression of THBS4 is increased in resected PCa tissue samples. In the present study, a microarray containing 8,277 IncRNA probes and 32,207 mRNA probes was used to identify dysregulated mRNAs in three patients with PCa. Among these mRNAs, 354 were significantly upregulated and 350 were significantly downregulated $(\mathrm{P}<0.05 ; \mathrm{FC}>2$; Fig. 1A). The most significantly upregulated mRNA was THBS4 $(\mathrm{P}<0.05 ; \mathrm{FC}>2)$.

THBS4 was selected to confirm the differential expression levels in 46 paired $\mathrm{PCa}$ and adjacent non-tumor tissue samples using RT-qPCR. The expression of THBS4 was significantly higher in the PCa tissues, compared with that in the adjacent non-tumor tissues (Fig. 1B).

Similarly, the results of the Western blot analyses revealed that the protein expression level ofTHBS was higher in the PCa tumor tissues, compared with the adjacent non-tumor tissues collected from the same patients (Fig. 1C). 
A

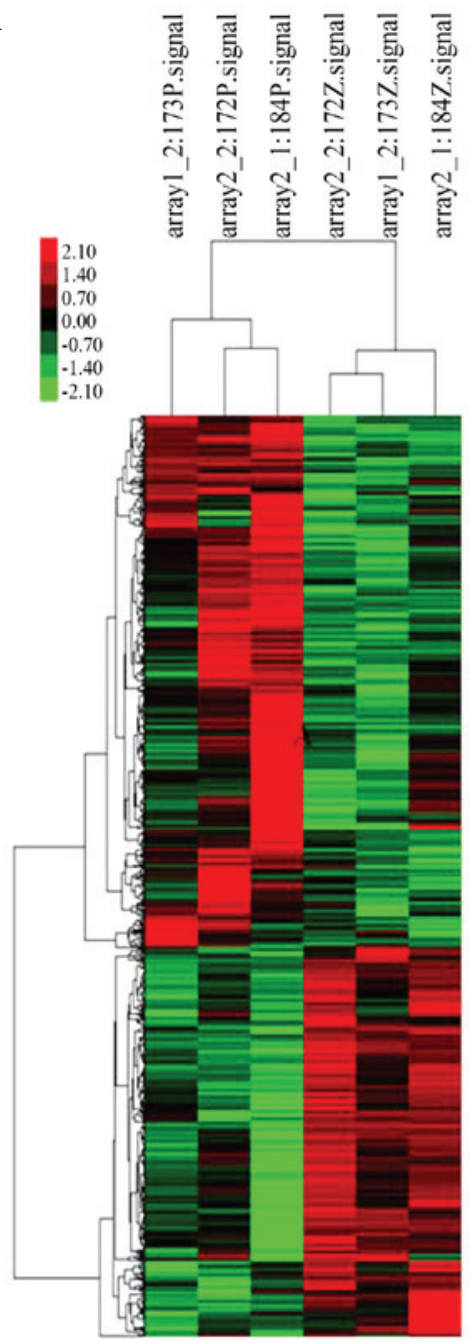

B

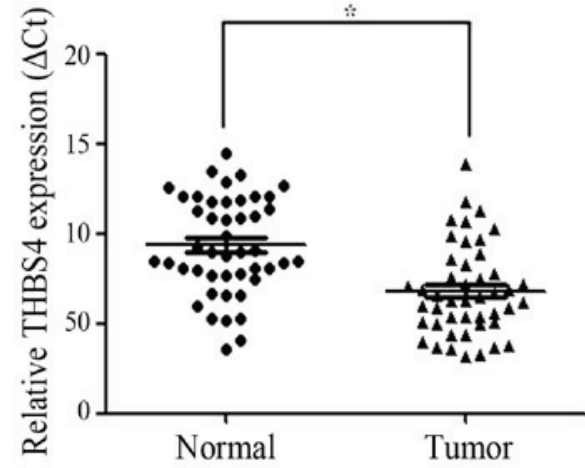

C

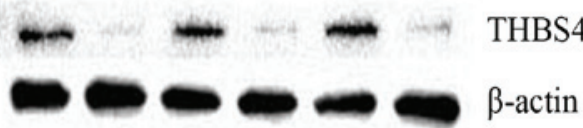

$\begin{array}{lllllllll}\mathrm{T} 1 & \mathrm{~N} 1 & \mathrm{~T} 2 & \mathrm{~N} 2 & \mathrm{~T} 3 & \mathrm{~N} 3\end{array}$

D

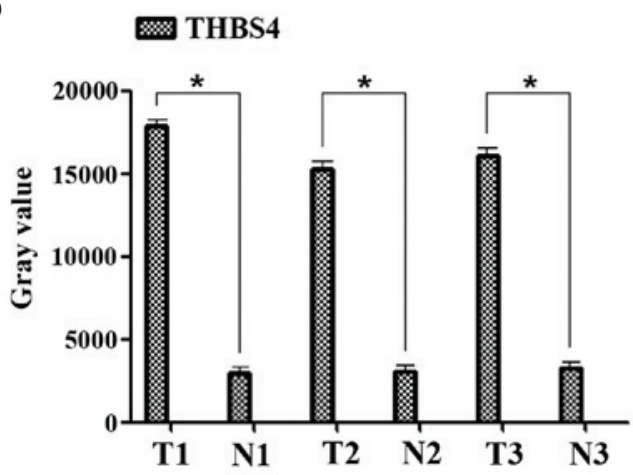

Figure 1. Expression of 1ncRNA-THBS4-003 in PCa and adjacent non-tumor tissues. (A) A microarray containing 8,277 lncRNA probes and 32,207 mRNA probes was used to identify dysregulated mRNAs in three patients with PCa. Of these, $354 \mathrm{mRNAs}$ were significantly upregulated and 350 were significantly downregulated $(\mathrm{P}<0.05 ; \mathrm{FC}>2$; red $=$ high expression, green $=$ low expression). (B) Higher expression levels of THBS4 were found in PCa tissues, compared with adjacent non-tumor tissues. (C) Protein expression levels of THBS4 in three matched non-tumor/tumor tissues were detected using Western blot analysis (D) Quantification of western blot analysis. Data are presented as the mean \pm standard deviation. ${ }^{*} \mathrm{P}<0.05$. $\beta$-actin was used as an internal control. PCa prostate cancer; THBS4, thrombospondin 4; N, non-tumor; T, tumor.

Expression of IncRNA-THBS4-003 is increased in resected PCa tissue samples. The expression of IncRNA-THBS4-003 was analyzed in 46 primary $\mathrm{PCa}$ and adjacent non-tumor tissue samples using RT-qPCR. The expression of IncRNA-THBS4-003 was significantly higher in the tumor tissues, compared with non-tumor tissues (Fig. 2A).

In addition, patients with Gleason scores $>7$ exhibited higher expression levels of IncRNA-THBS4-003, compared with the patients with lower Gleason scores (Fig. 2B).

Statistical analyses of the expression levels of THBS4 and lncRNA-THBS4-003 revealed a Pearson's correlation coefficient of $0.641(\mathrm{P}<0.0001)$, indicating a positive correlation between the expression levels of THBS4 and lncRNA-THBS4-003 in PCa (Fig. 2C).

Effects of THBS4 knockdown on PCa cell lines. In the present study, siRNA-mediated knockdown of THBS4 was performed in the PCa cells to investigate the effects of THBS4 on the migration and invasion of the PCa cells. The level of silencing achieved was also analyzed by performing RT-qPCR and
Western blotting. The mRNA expression levels of THBS4 and IncRNA-THBS4-003 were successfully reduced in the cells transfected with si-THBS4 (Fig. 3A). Suppressing the expression of THBS4 also reduced the expression levels of p38 and MMP-9 (Fig. 3B). Furthermore, the numbers of migrated and invaded cells transfected with si-THBS4 were significantly lower, compared with the cells transfected with the control (Fig. 3C and D).

Knockdown of IncRNA-THBS4-003 inhibits PCa cell migration and invasion in vitro. As shown in Fig. 4A, the expression levels of lncRNA-THBS4-003 in the PC-3 cells transfected with siRNA were examined. IncRNA-THBS4-003 knockdown significantly suppressed the migratory and invasive abilities of the PCa cells (Fig. 4B and C).

Reciprocal regulation of IncRNA-THBS4-003 and THBS4 contribute to $P C$ a cell line migration and invasion, and regulate levels of MMP-9 through the MAPK signaling pathway. Knocking down the expression of THBS4 successfully 
A

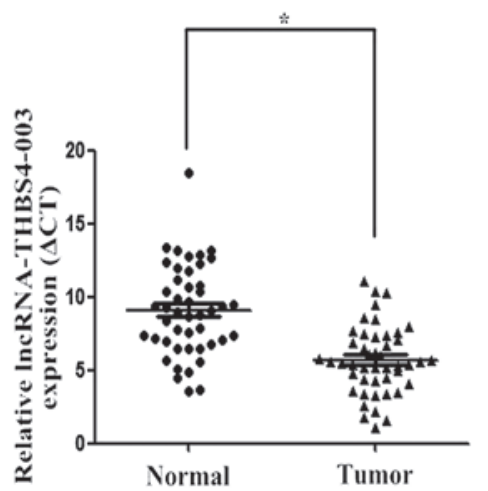

B

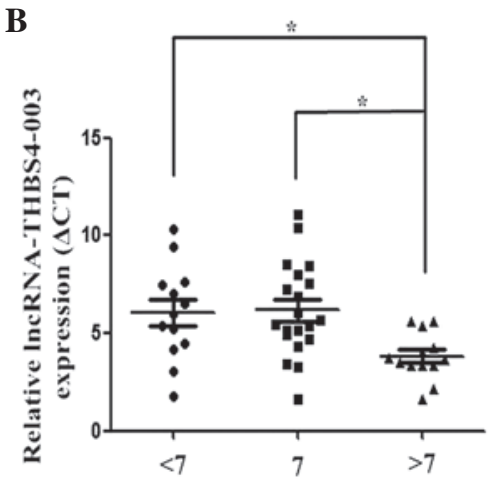

C

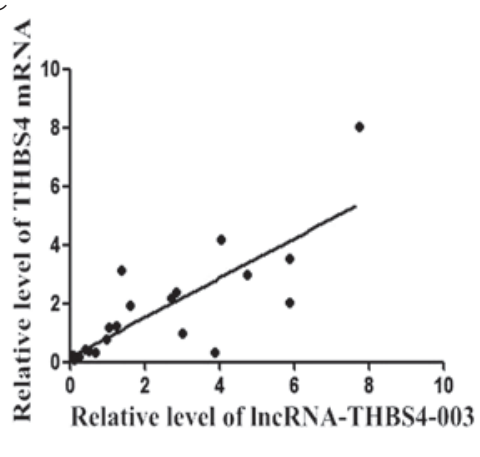

Figure 2. Expression of 1ncRNA-THBS4-003 in PCa and adjacent non-tumor tissues. (A) Expression levels of lncRNA-THBS4-003 were higher in the PCa tissues, compared with the adjacent non-tumor tissues. (B) Expression of lncRNA-THBS4-003 in patients with different Gleason scores. $\beta$-actin was used as a loading control. Data are presented as the mean \pm standard error of the mean. ${ }^{*} \mathrm{P}<0.05$ (determined using Student's $t$-test). (C) Expression levels of THBS4 and lncRNA-THBS4-003 were positively correlated in PCa tissues ( $\mathrm{P}<0.0001)$. PCa, prostate cancer; lncRNA, long noncoding RNA; THBS4, thrombospondin 4.

A

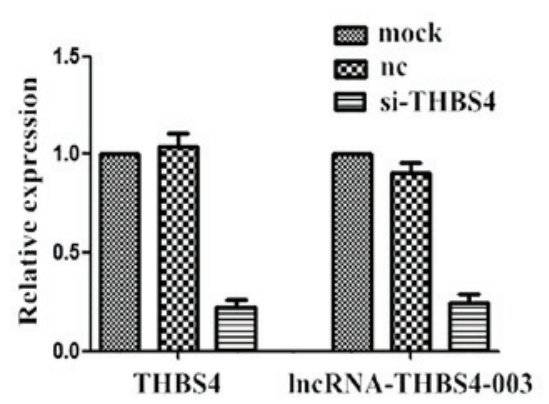

C
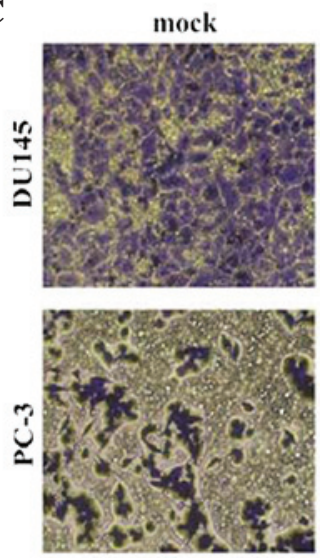

D
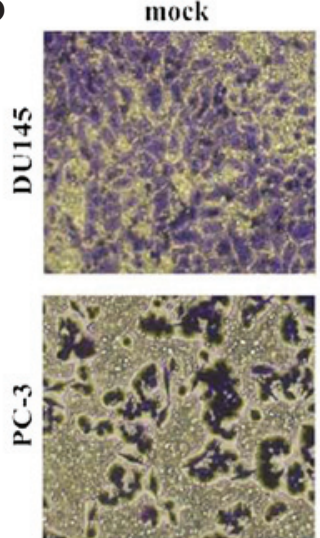

B

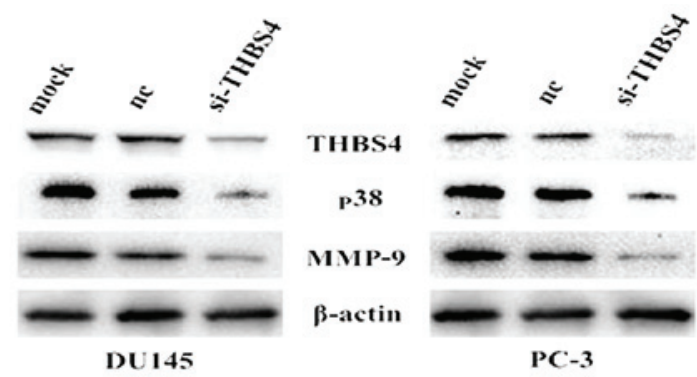

si-THBS4
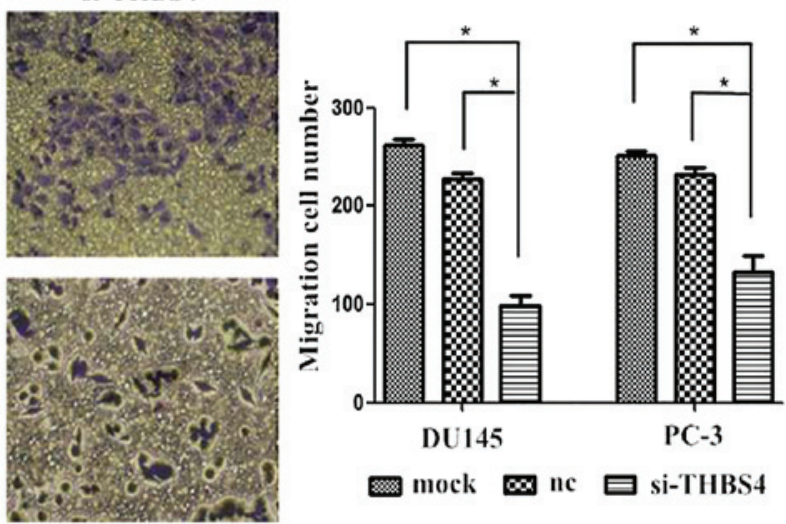

si-THBS4
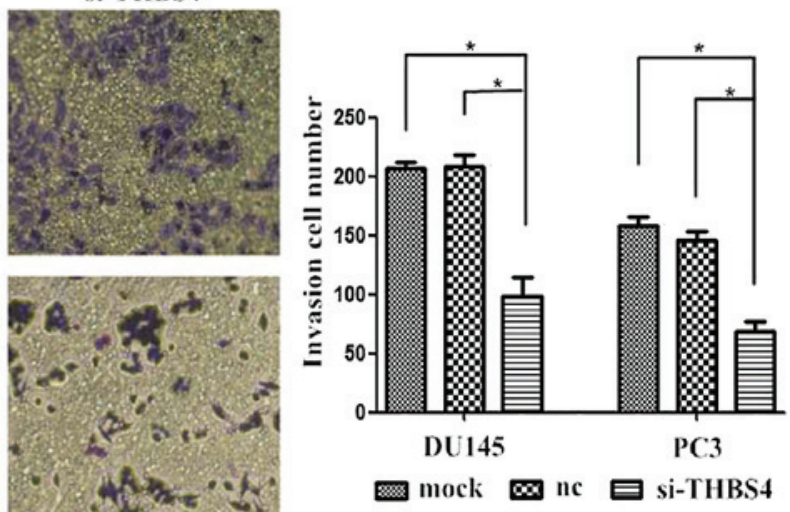

Figure 3. Effects of THBS4 knockdown on PCa cell lines. (A) Expression levels of THBS4 mRNA and lncRNA-THBS4-003 following transfection with mock, nc or si-THBS4. $\beta$-actin was used as a loading control. (B) Expression levels of THBS4, P38 and MMP-9 following transfection with mock, nc or si-THBS4. THBS4 knockdown inhibited the cell (C) migration and (D) invasion in vitro. Data are presented as the mean \pm standard error of the mean of at least three independent experiments. "P<0.05. Original magnification x200. PCa, prostate cancer; lncRNA, long noncoding RNA; THBS4, thrombospondin 4; si-THBS4, small interfering THBS4; MMP-9, matrix metallorpoteinase-9; nc, negative control. 
A

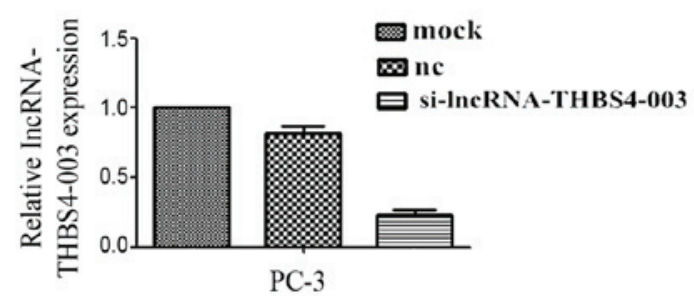

B
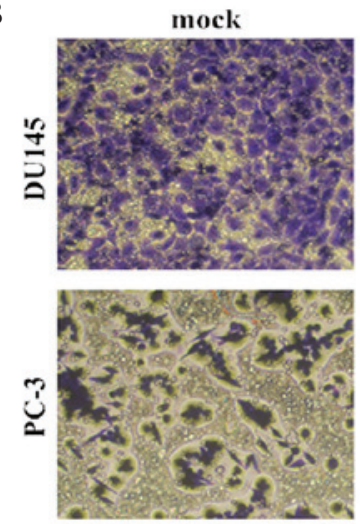

C
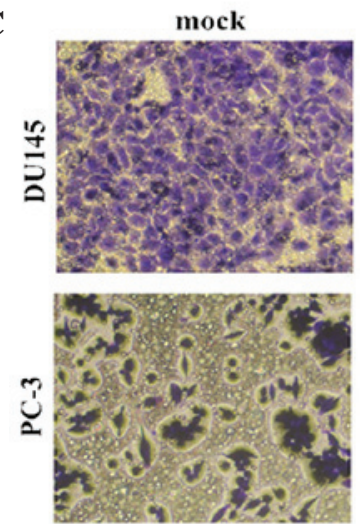

ne
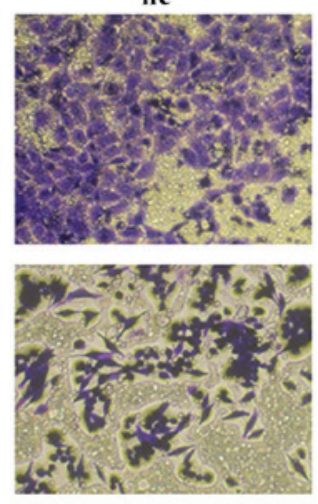

nc
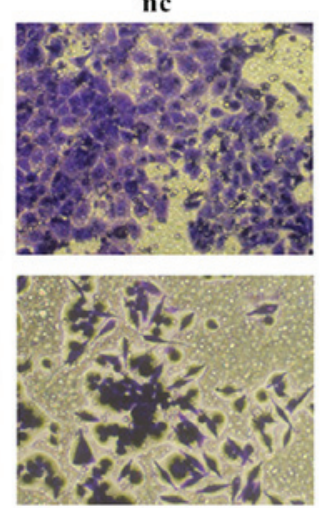

si-IncRNA-THBS4-003
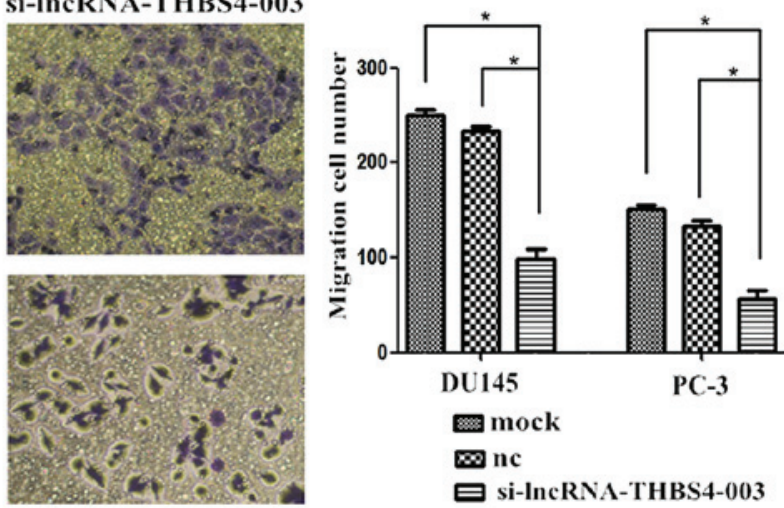

si-IncRNA-THBS4-003
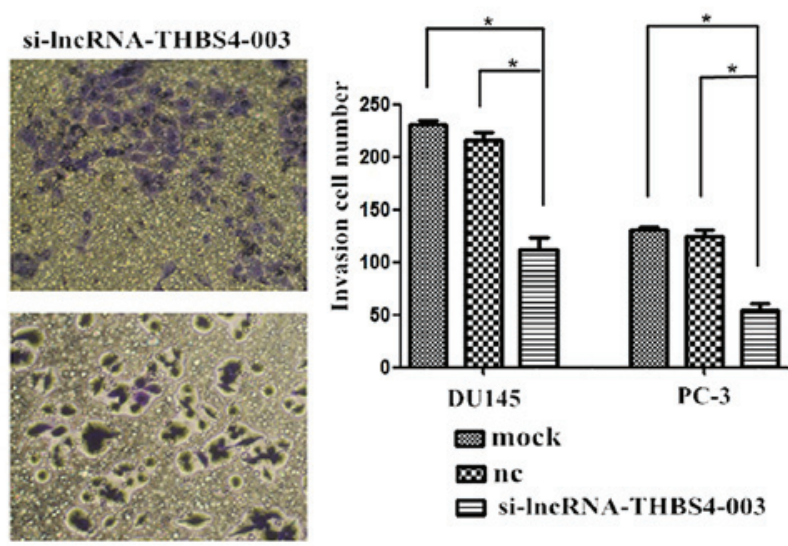

Figure 4. Knockdown of lncRNA-THBS4-003 inhibits PCa cell line migration and invasion in vitro. (A) Expression levels of lncRNA-THBS4-003 in transfected PC-3 cells were measured using reverse transcription-quantitative polymerase chain reaction analysis. $\beta$-actin was used as a loading control (determined using Student's $t$-test). 1ncRNA-THBS4-003 knockdown inhibited cell (B) migration and (C) invasion in vitro. Data are presented as the mean \pm standard error of the mean of at least three independent experiments. " $\mathrm{P}<0.05$. Original magnification $\mathrm{x} 200$. PCa, prostate cancer; lncRNA, long noncoding RNA; THBS4, thrombospondin 4; si-THBS4, small interfering-THBS4, nc, negative control.

reduced the expression of IncRNA-THBS4-003 in the cells transfected with si-THBS4 (Fig. 3A). Statistical analyses of the expression levels of THBS4 and lncRNA-THBS4-003 found a Pearson's correlation coefficient of 0.641 ( $\mathrm{P}<0.0001$; Fig. 2C). At $72 \mathrm{~h}$ post-transfection, the protein levels of THBS4, p38 and MMP-9 were significantly decreased in the cells transfected with si-lncRNA-THBS4-003, compared with the cells transfected with the control siRNA (Fig. 5).

\section{Discussion}

The present study is the first, to the best our out knowledge, to report IncRNA-THBS4-003 as a potentially useful biomarker for disease progression in patients with PCa.

In the present study, a microarray containing 8,277 lncRNA probes and 32,207 mRNA probes was used to identify dysregulated mRNAs in three patients with PCa. A total of 354 mRNAs were found to be significantly upregulated and 350 were downregulated $(\mathrm{P}<0.05 ; \mathrm{FC}>2)$. The most significantly upregulated mRNA was THBS4 $(\mathrm{P}<0.05$; FC $>2)$. IncRNA-THBS4-003 is located at chromosome $5 \mathrm{p} 14.1$ and partially overlaps the protein-coding gene, THBS4. Western blot and RT-qPCR analyses revealed that lncRNA-THBS4-003 and the expression levels of THBS4 were higher in tumor tissues, compared with the adjacent non-tumor tissues collected from the same patients. Statistical analyses of THBS4 and lncRNA-THBS4-003 demonstrated a Pearson's correlation coefficient of $0.641(\mathrm{P}<0.0001)$. The expression level of 1ncRNA-THBS4-003 was significantly higher, compared with the adjacent non-tumor tissues. Patients with Gleason scores $>7$ exhibited higher expression levels of lncRNA-THBS4-003, compared with those with lower scores. The aberrant expression and function of lncRNA-THBS4-003 in PCa remain to be elucidated. Using cell migration and invasion assays to evaluate migratory and invasive responses, lncRNA-THBS4-003 knockdown was found to significantly 

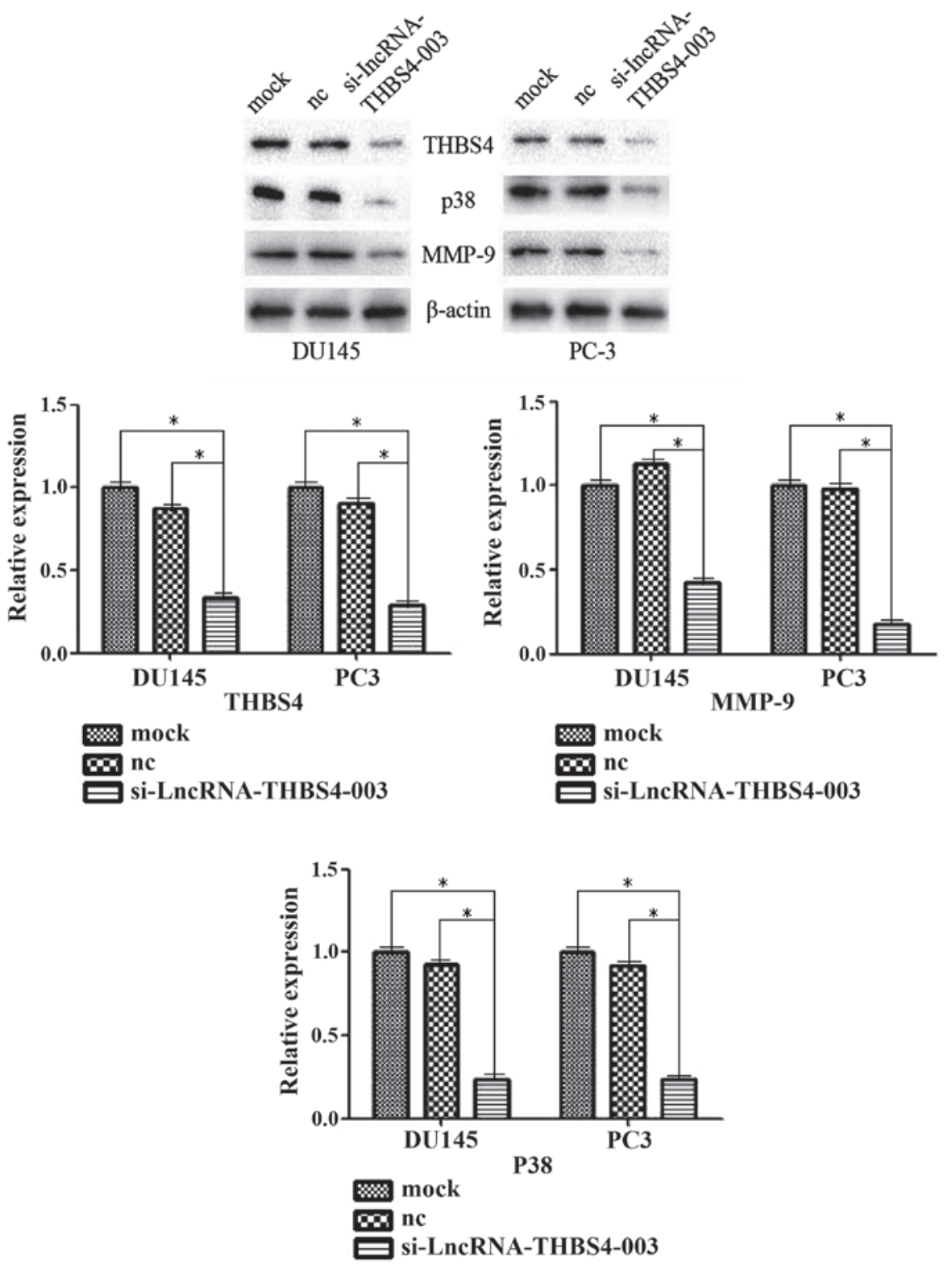

Figure 5. Expression levels of THBS4, P38 and MMP-9 are decreased following transfection with si-lncRNA-THBS4-003. "P<0.05. lncRNA, long noncoding RNA; THBS4, thrombospondin 4; si-THBS4, small interfering-THBS4; MMP-9, matrix metallorpoteinase-9; nc, negative control.

decrease the migratory and invasive abilities of the PCa cells in vitro, and to inhibit the expression levels of THBS4, p38 and MMP-9.

The thrombospondins (THBSs) are a family of five extracellular calcium-binding proteins (THBS1, THBS2, THBS3, THBS4 and THBS5/COMP), which are important in diverse processes through their interactions with the ECM. THBS4 is involved in several critical processes, including cellular proliferation, attachment, adhesion and migration, cytoskeletal organization, cell-to-cell interactions, and the promotion of neurite outgrowth (23-26). The role of THBS4 in cancer is well understood, and increasing evidence suggests that THBS4 is involved in colorectal, gastric and prostate carcinomas. THBS4 is reported to be expressed at high levels by cancer-associated fibroblasts as a constituent of desmoplastic stroma in prostate and gastric cancer (20,27-30).

Previous reports have shown that THBS4-induced activation of $\mathrm{p} 38$-MAPK regulates vascular inflammation and atherogenesis $(20,31,32)$. It is known that p38 MAPK is capable of regulating several cellular responses to cytokines and stress; however, studies have demonstrated that p38 is also closely associated with the development of different types of human cancer through its ability to elevate cancer cell migration and invasion in response to various stimuli, including inflammatory factors (31). Evidence has shown that p38 MAPK signals are involved in downregulating the expression of MMP-9, which has been linked to tumor migration and invasion (32).

The present study demonstrated significant downregulation in the protein levels of $\mathrm{p} 38$ and MMP-9 following the suppression of lncRNA-THBS4-003 or THBS4. The numbers of migrated and invaded cells transfected with si-IncRNA-THBS4-003 or si-THBS4 were also significantly lower, compared with the cells transfected with control siRNA.

The present study revealed that the forced knockdown of IncRNA-THBS4-003 or THBS4 decreased the in vitro migratory and invasive abilities of PCa cells through the MAPK signaling pathway. THBS4 is an adhesive glycoprotein that mediates cell-to-cell and cell-to-matrix interactions 
and is involved in the regulation of vascular inflammation. THBS4 and lncRNA-THBS4-003 can promote angiogenesis in prostate tissue (33). In the present study, p38 and MMP-9 were decreased following knockdown of THBS4 and lncRNATHBS4-003 in prostate cancer cell lines. MMP-9 can promote angiogenesis, tumor migration and invasion in prostate cancer. In conclusion, the present study demonstrates that THBS4 and lncRNA-THBS4-003 serve a significant role in PCa proliferation and migration via the MMP-9 and p38 MAPK signaling pathway.

\section{Acknowledgements}

This study was supported by the Priority Academic Program Development of Jiangsu Higher Education Institutions (grant no. JX10231801), the Program for Development of Innovative Research Team at the First Affiliated Hospital of Nanjing Medical University, the Provincial Initiative Program for Excellency Disciplines of Jiangsu Province (grant no. BL2012027), the National Natural Science Foundation of China (grant nos. 81201998 and 81372757) and the Natural Science Foundation of Jiangsu Province (grant no. BK20141495).

\section{References}

1. Jemal A, Siegel R, Ward E, Hao Y, Xu J, Murray T and Thun MJ: Cancer statistics, 2008. CA: Cancer J Clin 58: 71-96, 2008.

2. Zeigler-Johnson CM, Rennert H, Mittal RD, Jalloh M, Sachdeva R, Malkowicz SB, Mandhani A, Mittal B, Gueye SM and Rebbeck TR: Evaluation of prostate cancer characteristics in four populations worldwide. Can J Urol 15: 4056-4064, 2008.

3. Peyromaure EM, Mao K, Sun Y, Xia S, Jiang N, Zhang S, Wang G, Liu Z and Debré B: A comparative study of prostate cancer detection and management in China and in France. Can J Urol 16: 4472-4477, 2009.

4. Gibb EA, Brown CJ and Lam WL: The functional role of long non-coding RNA in human carcinomas. Mol Cancer 10: 38, 2011.

5. ENCODE Project Consortium, Birney E, Stamatoyannopoulos JA, Dutta A, Guigó R, Gingeras TR, Margulies EH, Weng Z, Snyder M, Dermitzakis ET, et al: Identification and analysis of functional elements in $1 \%$ of the human genome by the ENCODE pilot project. Nature 447: 799-816, 2007.

6. Yang L, Duff MO, Graveley BR, Carmichael GG and Chen LL: Genomewide characterization of non-polyadenylated RNAs. Genome Biol 12: R16, 2011.

7. Kapranov P, St Laurent G, Raz T, Ozsolak F, Reynolds CP, Sorensen PH, Reaman G, Milos P, Arceci RJ, et al: The majority of total nuclear-encoded non-ribosomal RNA in a human cell is 'dark matter' un-annotated RNA. BMC Biol 8: 149, 2010.

8. Martin L and Chang HY: Uncovering the role of genomic 'dark matter' in human disease. J Clin Invest 122: 1589-1595, 2012.

9. Fenoglio C, Ridolfi E, Galimberti D and Scarpini E: An emerging role for long non-coding RNA dysregulation in neurological disorders. Int J Mol Sci 14: 20427-20442, 2013.

10. Chen G, Wang Z, Wang D, Qiu C, Liu M, Chen X, Zhang Q, Yan G and Cui Q: LncRNADisease: A database for long-non-coding RNA-associated diseases. Nucleic Acids Res 41 (Database issue): D983-D986, 2013.

11. Rinn JL, Kertesz M, Wang JK, Squazzo SL, Xu X, Brugmann SA, Goodnough LH, Helms JA, Farnham PJ, Segal E and Chang HY: Functional demarcation of active and silent chromatin domains in human HOX loci by noncoding RNAs. Cell 129: 1311-1323, 2007.

12. Pibouin L, Villaudy J, Ferbus D, Muleris M, Prospéri MT, Remvikos Y and Goubin G: Cloning of the mRNA of overexpression in colon carcinoma-1: A sequence overexpressed in a subset of colon carcinomas. Cancer Genet Cytogenet 133: 55-60, 2002.

13. Fu X, Ravindranath L, Tran N, Petrovics G and Srivastava S: Regulation of apoptosis by a prostate-specific and prostate cancer-associated noncoding gene, PCGEM1. DNA Cell Biol 25: 135-141, 2006.
14. Calin GA, Liu CG, Ferracin M, Hyslop T, Spizzo R, Sevignani C, Fabbri M, Cimmino A, Lee EJ, Wojcik SE, et al: Ultraconserved regions encoding ncRNAs are altered in human leukemias and carcinomas. Cancer Cell 12: 215-229, 2007.

15. Lin R, Maeda S, Liu C, Karin M and Edgington TS: A large noncoding RNA is a marker for murine hepatocellular carcinomas and a spectrum of human carcinomas. Oncogene 26: 851-858, 2007.

16. Brody MJ, Schips TG, Vanhoutte D, Kanisicak O, Karch J, Maliken BD, Blair NS, Sargent MA, Prasad V and Malkentin JD: Dissection of thrombospondin-4 domains involved in intracellular adaptive endoplasmic reticulum stress-responsive signaling. Mol Cell Biol 36: 2-12, 2015.

17. da Silva HB, Amaral EP, Nolasco EL, de Victo NC, Atique R, Jank CC, Anschau V, Zerbini LF and Correa RG: Dissecting major signaling pathways throughout the development of prostate cancer. Prostate cancer 2013: 920612, 2013.

18. Birkedal-Hansen H, Moore WG, Bodden MK, Windsor LJ, Birkedal-Hansen B, DeCarlo A and Engler JA: Matrix metalloproteinases: A review. Crit Rev Oral Biol Med 4: 197-250, 1993.

19. Subramanian A and Schilling TF: Thrombospondin-4 controls matrix assembly during development and repair of myotendinous junctions. eLife 3: e02372, 2014.

20. Frolova EG, Pluskota E, Krukovets I, Burke T, Drumm C, Smith JD, Blech L, Febbraio M, Bornstein P, Plow EF and Stenina OI: Thrombospondin-4 regulates vascular inflammation and atherogenesis. Circ Res 107: 1313-1325, 2010.

21. Livak KJ and Schmittgen TD: Analysis of relative gene expression data using real-time quantitative PCR and the $2-\Delta \Delta \mathrm{Ct}$ method. Methods 25: 402-408, 2001.

22. Ding ZB, Shi YH, Zhou J, Shi GM, Ke AW, Qiu SJ, Wang XY, Dai Z, Xu Y and Fan J: Liver-intestine cadherin predicts microvascular invasion and poor prognosis of hepatitis B virus-positive hepatocellular carcinoma. Cancer 115: 4753-4765, 2009.

23. Narouz-Ott L, Maurer P, Nitsche DP, Smyth $\mathrm{N}$ and Paulsson M: Thrombospondin-4 binds specifically to both collagenous and non-collagenous extracellular matrix proteins via its C-terminal domains. J Biol Chem 275: 37110-37117, 2000.

24. Adams JC: Functions of the conserved thrombospondin carboxy-terminal cassette in cell-extracellular matrix interactions and signaling. Int J Biochem Cell Biol 36: 1102-1114, 2004.

25. Arber S and Caroni P. Thrombospondin-4, an extracellular matrix protein expressed in the developing and adult nervous system promotes neurite outgrowth. J Cell Biol 131: 1083-1094, 1995.

26. Stenina OI, Desai SY, Krukovets I, Kight K, Janigro D, Topol EJ and Plow EF: Thrombospondin-4 and its variants: Expression and differential effects on endothelial cells. Circulation 108: 1514-1519, 2003

27. Greco SA, Chia J, Inglis KJ, Cozzi SJ, Ramsnes I, Buttenshaw RL, Spring KJ, Boyle GM, Worthley DL, Leggett BA and Whitehall VL: Thrombospondin-4 is a putative tumour-suppressor gene in colorectal cancer that exhibits age-related methylation. BMC cancer 10: 494, 2010.

28. Förster S, Gretschel S, Jöns T, Yashiro M and Kemmner W: THBS4, a novel stromal molecule of diffuse-type gastric adenocarcinomas, identified by transcriptome-wide expression profiling. Mod Pathol 24: 1390-1403, 2011.

29. Dakhova O, Ozen M, Creighton CJ, Li R, Ayala G, Rowley D and Ittmann M: Global gene expression analysis of reactive stroma in prostate cancer. Clin Cancer Res 15: 3979-3989, 2009.

30. Gruel N, Lucchesi C, Raynal V, Rodrigues MJ, Pierron G, Goudefroye R, Cottu P, Reyal F, Sastre-Garau X and Fourquet A, et al: Lobular invasive carcinoma of the breast is a molecular entity distinct from luminal invasive ductal carcinoma. Eur J Cancer 46: 2399-2407, 2010.

31. Huang Q, Lan F, Wang X, Yu Y, Ouyang X, Zheng F, Han J, Lin Y, Xie Y, Xie F, et al: IL-1 $\beta$-induced activation of p38 promotes metastasis in gastric adenocarcinoma via upregulation of AP-1/c-fos, MMP2 and MMP9. Mol Cancer 13: 18, 2014.

32. Chien ST, Lin SS, Wang CK, Lee YB, Chen KS, Fong Y and Shih YW: Acacetin inhibits the invasion and migration of human non-small cell lung cancer A549 cells by suppressing the p38 $\alpha$ MAPK signaling pathway. Mol Cell Biochem 350: 135-148, 2011.

33. Frolova EG, Pluskota E, Krukovets I, Burke T, Drumm C, Smith JD, Blech L, Febbraio M, Bornstein P, Plow EF and Stenina OI: Thrombospondin-4 regulates vascular inflammation and atherogenesis. Circ Res 107: 1313-1325, 2010. 